\title{
BMJ Open Quality Reducing red blood cell folate testing: a case study in utilisation management
}

\author{
Ola Ismail, ${ }^{1}$ Ian Chin-Yee, ${ }^{2,3}$ Alan Gob, ${ }^{3}$ Vipin Bhayana, ${ }^{1,2}$ Angela Rutledge ${ }^{1,2}$
}

To cite: Ismail 0, Chin-Yee I, Gob A, et al. Reducing red blood cell folate testing: a case study in utilisation management. BMJ Open Quality 2019;8:e000531. doi:10.1136/ bmjoq-2018-000531

Received 13 September 2018 Revised 9 January 2019 Accepted 7 February 2019

\section{Check for updates}

C Author(s) (or their employer(s)) 2019. Re-use permitted under CC BY-NC. No commercial re-use. See rights and permissions. Published by BMJ.

${ }^{1}$ Pathology and Laboratory Medicine, Western University, London, Ontario, Canada ${ }^{2}$ Pathology and Laboratory Medicine, London Health Sciences Centre \& St. Joseph's Health Care London, London, Ontario, Canada

${ }^{3}$ Schulich School of Medicine, Western University, London, Ontario, Canada

Correspondence to Dr Angela Rutledge, Pathology and Laboratory Medicine, London Health Sciences Centre \& St. Joseph's Health Care London, London, Ontario, Canada;

angela.rutledge@|hsc.on.ca

\section{ABSTRACT}

Mandatory enrichment of wheat flour in Canada with folic acid since 1998 has caused folate deficiency to be rare. There were 3019 red blood cell (RBC) folate tests performed during an 18-month period at London Health Sciences Centre (LHSC)/St. Joseph's Healthcare London (SJHC) without any folate deficiency detected. We implemented a quality improvement initiative to reduce $\mathrm{RBC}$ folate testing at $\mathrm{LHSC} / \mathrm{SJHC}$. We began with a retrospective review of $\mathrm{RBC}$ folate tests performed during the previous 18 months. We identified physicians who had ordered more than five tests during this period and sent them an educational email to inform them of our intentions and solicit their input. We then discontinued RBC folate testing in-house and a pop-up window was introduced to the computerised physician order entry system stating that biochemist approval would be needed before samples would be sent out for testing. During the audited 18-month period, the average monthly test volume was 168 (SD 20). The three departments ordering the most RBC folate testing were nephrology (15\%), haematology $(7 \%)$ and oncology (7\%). Physician feedback was supportive of the change, and during the 2 months after targeted email correspondence, the average monthly test volume decreased $24 \%(p<0.01)$ to 128 (SD 1). On discontinuation of the test in-house and implementation of the pop-up, the average monthly test volume decreased another $74 \%(p<0.01)$ to 3 (SD 2). In the 10 months following discontinuation of the test on-site, there were only $39 \mathrm{RBC}$ folate tests performed with no deficiency detected. This initiative significantly reduced unnecessary RBC folate orders. The change in ordering on email contact suggests that physician education was an important factor reducing overutilisation. However, the most significant decrease came from restricting the test so that only orders approved by a biochemist would be performed.

\section{PROBLEM}

Pathology and Laboratory Medicine is the diagnostic services group providing laboratory testing for several hospitals belonging to the London Health Sciences Centre (LHSC) and St. Joseph's Healthcare London (SJHC) organisations. These hospitals provide tertiary care to the community in and around London, Ontario, serving a population of approximately 1.5 million.

While the amount of RBC folate testing performed annually at LHSC/SJHC has been dropping (8258 tests in 2013; 7580 tests in 2014; 4297 tests in 2015 and 2598 tests in 2016), the number of orders remained significant given the infrequency of folate deficiency. It was felt to be a poor use of laboratory resources to continue to allow unrestricted ordering of this test. We anticipated that implementation of ordering restrictions (necessity for biochemist approval) would reduce test volumes for RBC folate to such a substantial degree that it would no longer make sense to perform the testing on-site at LHSC/SJHC and so we determined that we would also refer the test to another laboratory for testing.

\section{BACKGROUND}

Folate, also known as vitamin B9, is a water-soluble vitamin that has important roles as a co-factor in a number of one-carbon transfer reactions. ${ }^{1}$ Folate is not synthesised by the body. It is mainly derived from folate-rich foods (eg, dark green leafy vegetables and lentils), fortified foods or vitamin supplements. ${ }^{2}$

WHO's suggestion for defining folate deficiency is a serum folate concentration $<10 \mathrm{nmol} / \mathrm{L}$ or an RBC folate concentration $<340 \mathrm{nmol} / \mathrm{L}$, based on the concentration at which the metabolite homocysteine becomes elevated. ${ }^{3}$ Severe folate deficiency causes macrocytic anaemia as it disrupts DNA synthesis. ${ }^{145}$ A low folate level early in pregnancy has been shown to be a risk factor for pregnancy complications such as abruptio placenta, eclampsia and congenital malformation and neural tube defects in infants. ${ }^{6-8}$ Also, low folate levels have been associated with diverse conditions such as cancer, ${ }^{9}$ cardiovascular disease, ${ }^{10}$ neurological disorders ${ }^{11}$ and osteoporosis. ${ }^{12}$ Risk factors for folate deficiency include inadequate food intake, heavy alcohol use, malabsorption (eg, due to coeliac or inflammatory bowel disease), folate depletion medication (eg, methotrexate, phenytoin) and pregnancy. ${ }^{1314}$

In 1998, the US Food and Drug Administration and Health Canada mandated the enrichment of all wheat flour with folic acid 
at an average daily intake of $100 \mu \mathrm{g} .{ }^{15-17}$ This mandatory fortification is now effective in 53 countries globally. Following fortification, the prevalence of folate deficiency was determined by several studies to be rare, averaging $<1 \%{ }^{18-21}$

Measurement of serum folate reflects recent dietary intake, whereas measurement of RBC folate reflects longterm folate tissue stores. ${ }^{22}$ Folate measurement in either serum or RBCs is usually performed by a competitive assay using folate-binding protein. For serum, no pretreatment steps are necessary. However, measurement of RBC folate first requires haematocrit to be measured and then requires a manual step to lyse the RBCs and release the folate. Following measurement of the folate level in the lysate, the result is normalised to the haematocrit. ${ }^{23} 24$ These steps cause RBC folate to be a more expensive and labour-intensive test. Despite these disadvantages of RBC folate over serum folate, many clinicians still prefer RBC folate since it is less susceptible to short-term changes in dietary folate intake. ${ }^{1}$ However, the best test for assessing folate status is still uncertain. ${ }^{1424-26}$

Numerous studies have shown that folate testing should be significantly reduced or discontinued. ${ }^{20}$ 27-33 Macrocytic anaemia, or even other forms of anaemia where there is an increased RBC distribution width, is felt to be one justifiable reason to order folate testing. However, links between anaemia and folate deficiency are not being observed since the introduction of folic acid fortification. ${ }^{28} 30$

Despite what is commonly felt to be excessive folate testing in countries that have fortification of flour with folic acid, there are few published quality improvement projects focused on reducing folate testing. In 2013, Health Quality Ontario-The Ontario Health Technology Advisory Committee decided to limit eligibility for coverage of RBC folate testing at private laboratories to patient populations with a) low haemoglobin levels and a high mean corpuscular volume (MCV); or b) suspected gastrointestinal disorders causing malabsorption or suspected malnutrition of any cause. ${ }^{31}$ In 2014 , Eaton et al implemented a non-intrusive clinical decision support tool in their hospital's electronic health record stating the low frequency of folate deficiency in the American population and suggesting that folate testing not be ordered, but they saw no significant effect on ordering practices. ${ }^{32}$ MacMillan et $a l^{33}$ found that by removing RBC folate from the list of tests available to order in their CPOE system for all physicians except haematologists or gastroenterologists, they were able to reduce ordering of the test by $94 \%$. Physicians other than haematologists or gastroenterologists were still able to order the test using a paper requisition or by calling the laboratory. Given the rarity of folate deficiency, we undertook a quality improvement initiative to decrease RBC folate testing at LHSC/SJHC.

\section{MEASUREMENT}

While measurement of RBC folate occurred at LHSC/ SJHC, haematocrit was calculated from impedance-based measurements of RBC count and MCV on a Beckman Coulter DXH 800, and folate was measured on a Roche e602 analyzer via a competitive electrochemiluminescent method. The reference interval for RBC folate was 1049-2941 nmol/L. Once RBC folate testing was referred out to another facility, the haematocrit was still measured at LHSC/SJHC, but the folate was measured on a Roche e411 analyzer, with a reference interval of 1150$2300 \mathrm{nmol} / \mathrm{L}$. Because this referral site discontinued their RBC folate test after about 8 months, we began sending samples to a new referral site. The new site was measuring folate on a Roche e411 analyzer, with a reference interval of $>1475 \mathrm{nmol} / \mathrm{L}$.

To collect our baseline data, we audited all RBC folate tests performed at LHSC/SJHC from both inpatients and outpatients between 1 October 2015 and 31 March 2017. There were 3019 tests performed with no results below LHSC/SJHC's reference interval or that would meet the WHO's definition of folate deficiency.

RBC folate data were also audited from 1 April 2017 to 30 April 2018, so that we could see the effects of emailing physicians at the end of March 2017, and of implementing the biochemist-approval process and beginning to refer out the test on 15 June 2017.

The audited data were analysed using Excel's PivotTable tool to determine the number of tests ordered by each physician, the departments ordering the most tests and monthly test volumes. Average monthly test volumes were calculated for the preintervention period (period 1 ), for period 2 after emailing physicians and for period 3 after implementing the biochemist-approval/referred out process. Because implementation of the utilisation restrictions took place on 15 June 2017, in the middle of a month, the month of June was omitted from the statistical analysis. The statistical process control X-MR chart was plotted using QI Macros SPC Software. Per cent difference in average monthly test volumes was calculated for period 2 compared with period 1 and for the difference between periods 2 and 3 compared with period 1 . A one-way analysis of variance (ANOVA) with a Tukey's post hoc test was performed on the monthly test volumes in periods 1,2 and 3 .

To examine the relationship between coeliac disease and folate deficiency, we audited the data for IgA antitissue transglutaminase (anti-tTG) antibodies and RBC folate between 22 May 2012 and 7 July 2016. We looked for patients with elevated anti-tTG, which would be consistent with coeliac disease, around the time (within about 2 months) that RBC folate was measured to see if any of the patients were deficient in folate. During the audited period, the anti-tTG test was an ELISA method from Euroimmun that was run on a Euroimmun Analyzer 1 platform. Results $\geq 20 \mathrm{RU} / \mathrm{mL}$ were considered to be positive for coeliac disease. 


\section{DESIGN AND STRATEGY}

First, we approached a city-wide diagnostic utilisation committee, a hospital group with broad representation of all specialties, diagnostic imaging and laboratories, with our proposal to reduce testing of RBC folate. The committee gave its support.

We then audited an 18-month period of RBC folate orders. From this audit, we identified 111 physicians who had ordered more than five tests. Those physicians received an email from the head of the Laboratory Medicine programme informing them of the infrequency of folate deficiency observed and our intentions to discontinue the test in-house and introduce a biochemist-approval process before samples would be sent to another laboratory for testing. The physicians were asked to provide feedback on whether they agreed with our proposal or had any objections. The chiefs of the departments these physicians belonged to were also emailed about the proposed changes and asked to notify their staff. Of the physicians who were contacted by email, there were 38 respondents. Thirty-four indicated that they supported the initiative and had no major concerns. Only four commented on the need for RBC folate testing in specific patient populations, such as coeliac disease, gastric bypass, severe anorexia or immigrants from countries without folic acid fortification. Physicians who raised concerns were reassured that the test would still be available, on biochemist approval, for patients at risk for folate deficiency.

After receiving stakeholder feedback and general support for the changes, we sent a memo to all physicians at LHSC/SJHC informing them of the upcoming changes.

On 15 June 2017, we discontinued the RBC folate test at LHSC/SJHC and introduced a pop-up window (figure 1) to the CPOE system within the hospital information system, Cerner Millennium, which would appear any time an order for RBC folate was entered.

Because regional hospitals use the same hospital information system and we did not have authority over those sites, a different pop-up window was created for those sites. This pop-up stated how infrequently folate deficiency was observed, but did not mention the need for biochemist-approval for testing.

The pop-up window created for LHSC/SJHC did not block orders for folate testing. We chose not to prevent orders from going into the CPOE system because we wanted to allow the order to go through for the cases where the physician would request biochemist approval. However, the physician could close the pop-up and proceed with the order for RBC folate without actually calling a clinical biochemist to request approval. We observed that a number of RBC folate samples were arriving at LHSC/SJHC laboratories for which biochemist approval had not been requested. Therefore, we implemented a policy whereby one of the clinical biochemists would check the pending $\log$ for RBC folate daily in the laboratory information system. For samples that had been received without approval, an email would be sent to the ordering physician that again emphasised the rarity of folate deficiency, stated that the test had been discontinued at LHSC/SJHC and informed the physician that he/she had 48 hours to request approval for the test or the order would be cancelled. For these samples that were received by the laboratory but did not have biochemist approval, the order would be cancelled 48 hours later and the sample that had been stored in the freezer would be discarded rather than being sent out for testing. However, the complete blood count (CBC) to obtain haematocrit would already have been performed on each sample received by the laboratory regardless of whether or not the folate order was cancelled.

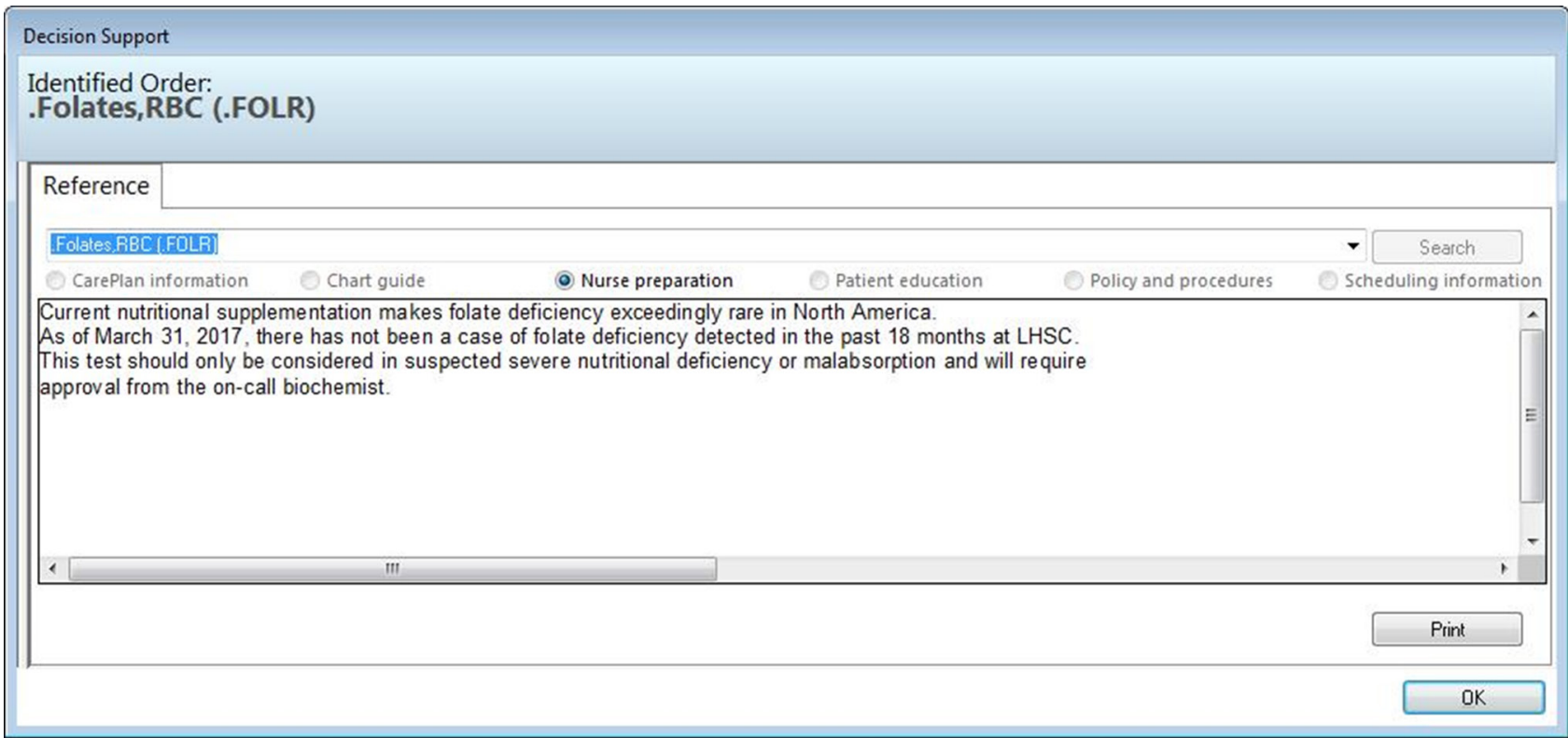

Figure 1 The pop-up window introduced to the computerised physician order entry system. 


$\left.\begin{array}{|c|c|}\text { Approached city-wide } \\ \text { utilization committee for } \\ \text { support for our initiative }\end{array}\right) \begin{aligned} & \text { Audited orders for RBC folate } \\ & \text { testing over an 18-month } \\ & \text { period to collect baseline data }\end{aligned}$

Physicians (111) who had ordered $>5$ RBC folate tests during the audited period, and the chiefs of the physicians' departments, were emailed; they were made aware of the lack of folate deficiency detected during the audited period, were informed of the proposed changes, and were asked if they agreed with or had any objections to the changes

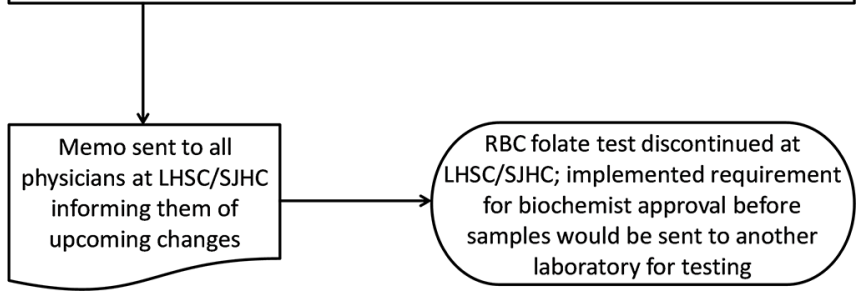

Figure 2 Summary of steps taken to reduce unnecessary RBC folate testing at LHSC/SJHC. LHSC, London Health Sciences Centre; RBC, red blood cell; SJHC, St. Joseph's Healthcare London.

Because a couple of the physicians contacted about the proposed changes raised concerns about patient populations with malabsorption, we attempted to gather evidence that these patients were unlikely to be folate deficient in an era of folic acid fortification. We audited data for RBC folate and anti-tTG antibodies, a screening test for coeliac disease, over a period of $>4$ years and looked for patients who had both tests performed.

The design for our quality improvement project is summarised in figure 2.

\section{RESULTS}

Figure 3A shows the monthly test volumes for RBC folate before and after the interventions. Figure $3 \mathrm{~B}$ shows how the average monthly test volume decreased in period 2 (the 2-month period following email contact with physicians who had ordered more than five tests during period 1 (preintervention) and the chiefs of the physicians' departments) compared with period 1 . There was a $24 \%$ decrease from 168 (SD 20) to 128 (SD 1) $(\mathrm{p}<0.01)$. Figure 3B also shows that the average monthly test volume decreased a further $74 \%$ in period 3 (the period following discontinuation of the in-house test and implementation of the requirement for biochemist approval for the send-out test) $(\mathrm{p}<0.01)$. Overall, there was a $98 \%$ decrease from 168 (SD 20) to 3 (SD 2) $(\mathrm{p}<0.01)$. Statistical process control demonstrated special cause variation beginning in June, corresponding to the intervention of restricting ordering and discontinuing the in-house test (figure 3A). Subsequent measurements indicated only common cause variation, suggesting a new, stable and better performing system.

During periods 1 and 2, there were 3019 and 255 RBC folate tests performed, respectively, and none of the results were below the reference interval for RBC folate or would meet the WHO's definition of folate deficiency. During

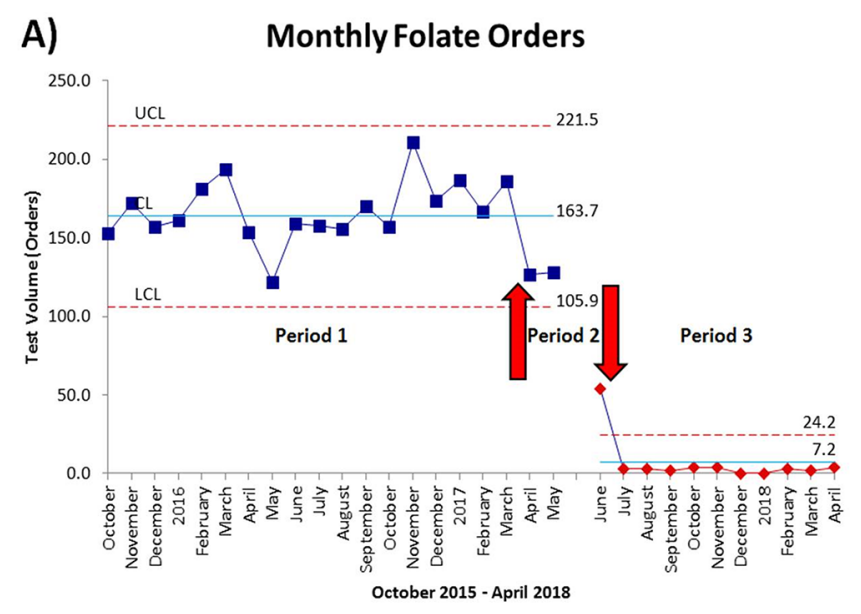

B)

\begin{tabular}{|c|c|c|c|}
\hline Period & $\begin{array}{c}\text { Average } \\
\text { Monthly Test } \\
\text { Volume (SD) }\end{array}$ & $\begin{array}{c}\text { P Value } \\
\text { Compared } \\
\text { to Period 1 }\end{array}$ & $\begin{array}{c}\text { P Value } \\
\text { Compared } \\
\text { to Period 2 }\end{array}$ \\
\hline 1) preintervention & $168(20)$ & & \\
\hline $\begin{array}{c}\text { 2) after emailing physicians } \\
\text { ordering }>5 \text { tests/year }\end{array}$ & $128(1)$ & $<0.01$ & $<0.01$ \\
\hline $\begin{array}{c}\text { 3) after discontinuation of } \\
\text { the test in-house and } \\
\text { implementation of } \\
\text { biochemist-approval process }\end{array}$ & $3(2)$ & $<0.01$ & \\
\hline
\end{tabular}

Figure 3 (A) Monthly red blood cell (RBC) folate test volumes for period 1 prior to the intervention; period 2 after emailing physicians who had ordered more than five tests during period 1 and the chiefs of the physician's departments and period 3 after discontinuing the in-house test and implementing a biochemist-approval process for the send-out test. (B) Average monthly test volumes for each period and comparison of periods 1,2 and 3 by a one-way analysis of variance with a Tukey's post hoc test. The month of June 2017 was omitted from the analysis because the discontinuation of the in-house test and implementation of the biochemist-approval process for the send-out test occurred in the middle of the month on 15 June 2017.

period 3, there were $25 \mathrm{RBC}$ folate tests performed. With the change of the referred out testing site after 8 months to one with a higher reference interval, there were two results below the reference interval, but none would meet the WHO's definition of folate deficiency.

Prior to the intervention, the three departments ordering the most tests were nephrology (15\%), haematology $(7 \%)$ and oncology $(7 \%)$. During the 10 months since the RBC folate test was discontinued at LHSC/ SJHC, the 39 RBC folate tests performed at referral laboratories were ordered by multiple departments, with oncology (13\%), haematology $(8 \%)$ and paediatric medicine $(8 \%)$ ordering the most tests.

We identified 11 patients who had anti-tTG antibody levels elevated above the cut-off for coeliac disease at approximately the same time (within about 2 months) RBC folate was measured, and none would meet the WHO's definition of folate deficiency.

In terms of cost, the total cost for reagents, labour, supplies and the instrument for measurement of $\mathrm{RBC}$ folate when the test was performed at LHSC/SJHC was 
about $\$ C 6.80 /$ test, not including the cost of the CBC. At an average monthly test volume of 168 prior to the intervention, the cost would be approximately $\$$ C1140. The cost to refer out RBC folate testing to another facility is $\$ \mathrm{C} 20 /$ sample, which includes the shipping costs. At an average monthly test volume of 3 since the test was referred out and the biochemist-approval process was implemented, the monthly cost has been decreased about $95 \%$ to approximately $\$$ C60.

\section{LESSONS AND LIMITATIONS}

In our study, we saw that an email informing physicians (and their chiefs) about the proposed changes and their rationale, as well as asking for feedback, reduced RBC folate ordering by about $24 \%$ in the subsequent 2 months. While the drop in orders following email contact with physicians was statistically significant according to a one-way ANOVA with a Tukey's post hoc, it was not associated with special cause variation. Perhaps if we had allowed period 2 to continue for $>2$ months, special cause variation would have been seen. Since it was not seen, we cannot be certain that the drop in ordering during those 2 months was not due to chance. It is also not clear how long this decrease in ordering would have been sustained if that had been our only intervention. As demonstrated in a previous study by Eaton et $a l^{32}$ educational interventions such as email or a pop-up at the time of ordering are rarely sufficient to modify laboratory test ordering practices. In our project, while the larger decrease in RBC folate testing occurred as a result of implementing a biochemist-approval process, we believe that contacting stakeholders helped physicians to feel that they were being consulted and involved in the change process. Seeking feedback increased acceptance of the changes, which was critical to the process as physicians tend to have an aversion to pop-ups in the CPOE system and dislike any restrictions to the tests they are ordering. We believe that without the initial communication and educational steps, the later stages of our project would have been viewed as draconian.

The advantage of the biochemist-approval approach we employed is that it acknowledges that there may be rare instances where folate testing may be indicated, but functions as a hard stop for routine orders. If the physician feels that RBC folate testing is indicated, he/she must discuss the situation with the biochemist and justify the request for the sample to be sent out for testing. Unfortunately, while our method functions as a hard stop, it can be bypassed; our pop-up window can be ignored and the physician can proceed with the order and have the sample sent to the laboratory without ever contacting a clinical biochemist to request approval. Ten months after the implementation, a clinical biochemist is still checking the pending $\log$ in the laboratory information system daily to see which samples have been received without approval and then sending emails to the ordering physicians instructing that the order will be cancelled if biochemist approval is not sought within 48 hours. Most of these orders are subsequently cancelled. While the number of samples being received without approval is limited, this process remains time consuming with multiple emails being sent each week. This is in contrast to the original intention of placing the onus for obtaining biochemist approval on the ordering physician.

In addition, for the samples sent to the laboratory without biochemist approval being requested, the $\mathrm{CBC}$ (haematocrit) is still run regardless of whether a sample is sent out for folate testing or not. It is not clear how many of the physicians would have ordered the CBC even if the RBC folate test was not being ordered, but presumably some of these CBCs are being performed unnecessarily because our method does not stop unapproved RBC folate orders from going into the CPOE system.

A future change to decrease the time involved in managing unapproved orders is being considered. Given that the samples that have received biochemist approval to be sent out thus far have not identified any cases of folate deficiency, one approach would be to add a warning to the pop-up to indicate that the order will be cancelled unless the physician contacts a biochemist for approval. This approach would place the onus on the ordering physician and not the laboratory to seek approval. However, it would not stop unnecessary CBC measurements.

\section{CONCLUSION}

Our interventions, including targeted emails, an educational memo, a decision support pop-up in the CPOE system and ultimately implementing the requirement for biochemist-approval, successfully decreased RBC folate testing by approximately $98 \%$. Because our intervention of implementing biochemist-approval functions as a hard stop, this decrease will likely be sustained over time. There is little published literature on utilisation projects to reduce folate testing. Eaton $e t a \hat{l}^{2}$ found their non-intrusive clinical decision support tool in the hospital's electronic health record was ineffective at reducing folate testing. MacMillan $e t a l^{33}$ found their approach of restricting ordering of $\mathrm{RBC}$ folate testing in the $\mathrm{CPOE}$ system to haematologists or gastroenterologists reduced orders by $94 \%$. We present a different approach that did not give privileges for folate ordering to any specialty. Other sites would be able to use a similar approach to ours. If a clinical biochemist is not available at the location, test approvals could be managed by another individual who is willing to take on the responsibility.

MacMillan $e t a \vec{l}^{3}$ estimated that their intervention of removing RBC folate from the CPOE system for all physicians except haematologists or gastroenterologists saved over \$C280 000 in 3 years. Their test volumes prior to the intervention were considerably higher than ours and their estimated cost per test (\$C14) was more than double ours. While our calculated savings are not nearly as large, it does appear that our project is saving some healthcare dollars from being spent on unnecessary testing. The 
funds and time no longer being spent by LHSC/SJHC on RBC folate testing may be helpful in allowing new tests to be developed and implemented. Or, as there are often cuts to the laboratory budget, reducing money spent on tests that add little value, such as RBC folate, may allow the laboratory to continue to perform current tests that do add value.

We did not have enough data to thoroughly examine the relationship between coeliac disease and folate deficiency. However, the fact that we have seen such little folate deficiency over the years, when RBC folate measurements would have been performed in patients with active coeliac disease, severe anorexia, malnutrition or malabsorption due to a variety of other causes, suggests that even these patients are not likely to be folate deficient. Folate deficiency has not been well studied in patients with these conditions since folic acid fortification came into effect.

A common reason for folate testing is the finding of macrocytic anaemia. A number of studies have shown that in countries with folic acid fortification, most patients with folate deficiency do not have macrocytosis and vice versa. ${ }^{18-20} 28$ Of note, prior to the implementation of RBC folate ordering restrictions by MacMillan et $a l^{33}$ haematologists were the most frequent ordering specialty as part of their workup for macrocytic anaemia and this group was exempted from the changes implemented to folate ordering. As folate deficiency is no longer a common cause of macrocytic anaemia, this commonly taught algorithmic approach to macrocytic anaemia should be revised and specific exemptions regarding folate ordering are unlikely to be necessary for any subspecialty in North America.

Most of the reasons that biochemist approval was sought and given in our study were malnutrition, malabsorption and/or macrocytic anaemia. Even in these patients felt to be at increased risk of folate deficiency, folate deficiency was not observed. The data suggest that a permanent hard stop of not referring samples out for testing could be considered. However, there would likely be resistance from physicians until there is more evidence or clinical practice guidelines to support this course of action. Therefore, more thorough study of the likelihood of folate deficiency in patients with malnutrition, malabsorption or macrocytic anaemia in countries with folic acid fortification should be performed, followed by education of physicians on clinical indications that truly merit folate testing.

Contributors 0 l was involved in manuscript preparation. AR, IC-Y and VB contributed to project design, implementation, data analysis and manuscript preparation. AG advised on quality improvement methodology and constructed and interpreted the SPC chart.

Funding The authors have not declared a specific grant for this research from any funding agency in the public, commercial or not-for-profit sectors.

Competing interests None declared.

Patient consent for publication Not required.

Provenance and peer review Not commissioned; externally peer reviewed.
Open access This is an open access article distributed in accordance with the Creative Commons Attribution Non Commercial (CC BY-NC 4.0) license, which permits others to distribute, remix, adapt, build upon this work non-commercially, and license their derivative works on different terms, provided the original work is properly cited, appropriate credit is given, any changes made indicated, and the use is non-commercial. See: http://creativecommons.org/licenses/by-nc/4.0/.

\section{REFERENCES}

1. Farrell CJ, Kirsch SH, Herrmann M. Red cell or serum folate: what to do in clinical practice? Clin Chem Lab Med 2013;51:555-69.

2. Shane B. Folate status assessment history: implications for measurement of biomarkers in NHANES. Am J Clin Nutr 2011;94:337S-42.

3. de Benoist B. Conclusions of a WHO technical consultation on folate and vitamin B12 deficiencies. Food Nutr Bull 2008;29(2 Suppl):S238-S244.

4. Izak G, Rachmilewitz M, Zan S, et al. The effect of small doses of folic acid in nutritional megaloblastic anemia. Am J Clin Nutr 1963;13:369-77.

5. Tisman G. diagnostic value of serum folate level. N Engl J Med 1972;287:466-7.

6. Rothman D. Folic acid in pregnancy. Am J Obstet Gynecol 1970;108:149-75.

7. Davis BA, Bailey LB. Gregory JF 3rd, Toth FP, Dean J, Stevenson RE. Folic acid absorption in women with a history of pregnancy with neural tube defect. Am J Clin Nutr 1995;62:782-4.

8. Charles DH, Ness AR, Campbell D, et al. Folic acid supplements in pregnancy and birth outcome: re-analysis of a large randomised controlled trial and update of Cochrane review. Paediatr Perinat Epidemiol 2005;19:112-24.

9. Ulrich CM. Folate and cancer prevention: a closer look at a complex picture. Am J Clin Nutr 2007;86:271-3.

10. Moat SJ, Lang D, McDowell IF, et al. Folate, homocysteine, endothelial function and cardiovascular disease. $J$ Nutr Biochem 2004;15:64-79.

11. Djukic A. Folate-responsive neurologic diseases. Pediatr Neurol 2007;37:387-97.

12. Herrmann M, Widmann T, Herrmann W. Homocysteine-a newly recognised risk factor for osteoporosis. Clin Chem Lab Med 2005;43:1111-7.

13. Kaferle J, Strzoda CE. Evaluation of macrocytosis. Am Fam Physician 2009;79:203-8.

14. Gudgeon P, Cavalcanti R. Folate testing in hospital inpatients. Am J Med 2015;128:56-9.

15. Crider KS, Bailey LB, Berry RJ. Folic acid food fortification-its history, effect, concerns, and future directions. Nutrients 2011;3:370-84.

16. Canada H. Food and drug regulations, amendment schedule no. 1066. Ottawa: Health Canada 1997.

17. Food and Drug Administration. Food standards: amendment of standards of identity for enriched grain products to require addition of folic acid [Internet]. Federal Register 1996;61:8781-97.

18. Latif T, Hsi ED, Rybicki LA, et al. Is there a role for folate determinations in current clinical practice in the USA?. Clin Lab Haematol 2004;26:379-83.

19. Odewole OA, Williamson RS, Zakai NA, et al. Near-elimination of folate-deficiency anemia by mandatory folic acid fortification in older US adults: reasons for geographic and racial differences in stroke study 2003-2007. Am J Clin Nutr 2013;98:1042-7.

20. Joelson DW, Fiebig EW, Wu AH. Diminished need for folate measurements among indigent populations in the post folic acid supplementation era. Arch Pathol Lab Med 2007;131:477-80.

21. Ray JG, Vermeulen MJ, Boss SC, et al. Declining rate of folate insufficiency among adults following increased folic acid food fortification in Canada. Can J Public Health 2002;93:249-53.

22. Pietrzik K, Lamers Y, Brämswig S, et al. Calculation of red blood cell folate steady state conditions and elimination kinetics after daily supplementation with various folate forms and doses in women of childbearing age. Am J Clin Nutr 2007;86:1414-9.

23. Wright AJ, Finglas PM, Southon S. Erythrocyte folate analysis: saponin added during lysis of whole blood can increase apparent folate concentrations, depending on hemolysate $\mathrm{pH}$. Clin Chem 2000;46:1978-86.

24. Pillay TS, Oosthuizen NM. Why are we still measuring red cell folate instead of just serum folate? J Clin Pathol 2014;67:289.

25. Galloway M, Rushworth L. Red cell or serum folate? Results from the National Pathology Alliance benchmarking review. J Clin Pathol 2003;56:924-6. 
26. Owen WE, Roberts WL. Comparison of five automated serum and whole blood folate assays. Am J Clin Pathol 2003;120:121-6.

27. Theisen-Toupal J, Horowitz G, Breu A. Low yield of outpatient serum folate testing: eleven years of experience. JAMA Intern Med 2014;174:1696-7.

28. Shojania AM, von Kuster K. Ordering folate assays is no longer justified for investigation of anemias, in folic acid fortified countries. BMC Res Notes 2010;3:22.

29. Wu AH, Wu WH. Folate testing: time to retire your VCR. JAMA Intern Med 2014:174:1697-8.

30. Theisen-Toupal J, Horowitz GL, Breu AC. Utility, charge, and cost of inpatient and emergency department serum folate testing. J Hosp Med 2013;8:91-5.
31. Diagnostic Services and Planning Branch. Lab test utilization management changes to the schedule of benefits - laboratory services [Internet]. INFO Bulletin 2013 http://www.health.gov.on. ca/en/pro/programs/ohip/bulletins/4000/bul4584.pdf (cited 8 May 2018).

32. Eaton KP, Chida N, Apfel A, et al. Impact of nonintrusive clinical decision support systems on laboratory test utilization in a large academic centre. J Eval Clin Pract 2018;24:474-9.

33. MacMillan TE, Gudgeon P, Yip PM, et al. Reduction in unnecessary red blood cell folate testing by restricting computerized physician order entry in the electronic health record. Am J Med 2018;131:939-44. 